\title{
Development of a Combination Forced Oscillation - Spirometer Device
}

\author{
Vargha Talebi and Geoffrey N. Maksym \\ Dalhousie University, Halifax, Nova Scotia, Canada \\ vtalebi@yahoo.com,gmaksym@dal.ca
}

\begin{abstract}
We developed a novel device, called the oscillation spirometer (OS) that can track changes in respiratory system impedance (Zrs) and can provide standard measures of respiratory resistance and spirometry. Current devices do not easily track temporal variation in Zrs. The device consists of a self-actuated piston containing an airflow resistance. During spirometry mode, the screen mesh pneumotachograph (PT) is stationary while during oscillation modes the PT translates generating oscillatory pressure. The actuator position is monitored continuously by a laser position sensing detector. To facilitate the design process, we developed a computer simulation of the actuator including mechanical and electromechanical compartments. A second model was created to simulate the patient Zrs and the two models were coupled and used to optimize design parameters such as magnet size, stroke volume, and piston cross-section, in a recursive design process. The result was a portable device with optimal stroke volume for expected patient loads. Three different designs were considered for the airflow resistance (orifice, groove and screen mesh resistances), and were tested using mock-up models. The final screen mesh resistance was linear, with a 98\% lower $2^{\text {nd }}$ order nonlinearity term than the next best design, and met American Thoracic Society standards. The OS could be used as a hand-held spirometer, or as a mounted FOT device suitable for detecting respiratory system resistance (Rrs) and its variation. It therefore has the potential to have a substantial impact in the respiratory market.
\end{abstract}

\section{Introduction}

Asthma is a chronic lung disease involving airway inflammation and remodeling, and is associated with airways that tighten or constrict too easily and too much [1]. The characteristic bronchoconstriction of asthma is often associated with symptoms such as wheezing, coughing, shortness of breath, and chest tightness [2]. The prevalence of asthma in Canada has been increasing over the last 20 years and currently over 2.4 million Canadians have been diagnosed with asthma, making it the most prevalent chronic condition in Canada [3]. It is also most common during childhood and affects approximately $12 \%$ of Canadian children and $5 \%$ of adults [4].

A diagnosis of asthma is usually based on a patient's symptoms, medical history, physical examination, and measures of pulmonary function. Pulmonary function is measured with standard testing known as spirometry. During a typical spirometry manoeuvre a patient blows into a spirometer as forcefully and for as long as possible until as much air as possible is exhaled from the lungs. The spirometer measures the airflow and volume during the manoeuvre. The amount of air exhaled in the first second, known as the forced expiratory volume in 1 second (FEV1) is measured and is an indicator of obstruction.

Spirometry requires significant patient compliance and performance of a learned manoeuvre, and thus it is not possible to achieve accurate results in very young children. It is also difficult in some other populations such as paralyzed or infirm individuals [5]. Currently, there is no clinically accepted method for measurement of lung function in children under the age of 6 where the majority of asthma is first diagnosed [6-8]. In such patient populations, diagnosis is based on a physician's assessment of symptoms. However, an alternative method for lung function testing that requires no patient effort is the forced oscillation technique (FOT), which is beginning to be used clinically particularly in Europe.

FOT applies low-amplitude pressure oscillations at the patient's airway opening during spontaneous breathing and records the mechanical properties of the lung, namely respiratory system impedance (Zrs). Zrs is obtained from patient pressure and flow and is a complex quantity that includes both resistance and reactance according to equation 1.1.

$$
Z r s=R r s+j X r s
$$


The real part is the respiratory system resistance (Rrs) while the imaginary part is the respiratory system reactance (Xrs) [9]. Zrs is computed as

$$
\operatorname{Zrs}(f)=\frac{P(f)}{\dot{V}(f)}
$$

where $\mathrm{P}(\mathrm{f})$ and $\dot{\mathrm{V}}(\mathrm{f})$ are the Fast Fourier Transforms of pressure and flow respectively.

Measurements of Zrs have been obtained after airway dilation and constriction in a variety of respiratory diseases [10-12]. Essentially when Rrs is increased it is inferred that airway diameter is decreased. Recently, measurements of the variation in Rrs (SDRrs) over short term recordings of a few minutes have shown that SDRrs is increased in asthma and is a more sensitive indicator than mean Rrs and FEV1 of asthma [13, 14]. It is also reduced more by bronchodilator in children with asthma than healthy controls [15]. These results suggest that important information appears to be present in the measurement of Rrs variation, and that this may be useful in asthma diagnosis and possibly in guiding therapy.

\subsection{Current devices}

Currently, there are two main commercially available FOT devices. The basic design methodology behind these two devices is similar. Both perform spirometry and FOT using a parallel pathway approach, through the use of a trap door which is shut to allow pressure oscillations to travel to the patient's airway opening. During spirometry, this door is opened to allow flow to move freely through the system. Both devices use a pressure oscillation source like a loudspeaker cone with a separate flow resistive element across which pressure is measured. These devices also have similar dead space volumes of approximately $80 \mathrm{ml}$. The dead space is the space within a device in which a patient's breath is trapped and consequently re-breathed, and therefore dead space should be as low as possible. Larger dead space is not desired as it modifies patient breathing which can in turn alter Zrs.

The novel FOT device designed as described in this report is known as the oscillation spirometer (OS) because of it ability to record conventional spirometry parameters and forced oscillation mechanics. In addition to spirometry and FOT, the OS measures variability of airway caliber which has been established to be an important indicator of asthma and airway smooth muscle (ASM) activity [15]. The methodology behind the design of the OS also overcomes the limitations of speaker-based systems, through the use of a self-actuated piston (SAP). Using a SAP removes the need for parallel flow pathways and large loudspeakers. Thus, the OS can be compact and lightweight and could be used as a hand-held unit for spirometry.

\section{Methods}

We used an iterative design process by creating models of the OS actuator and patient Zrs to test the performance in silico and optimize the device size.

The actuator model (Figure 1) was separated into electrical (coil resistance and inductance with current and voltage limits) and mechanical (mass and static/dynamic friction with position limits) transfer functions within the nonlinear plant. Then, a patient load was simulated using a single compartment model for respiratory mechanics consisting of a resistance and elastance. Disturbance compensation was added to compensate for cylinder back pressure and a position feedback loop was incorporated for piston stability. An iterative process was used in which control parameter combinations (proportional derivative gain) were tested to achieve maximal rise time and minimal overshoot responses to a step input.

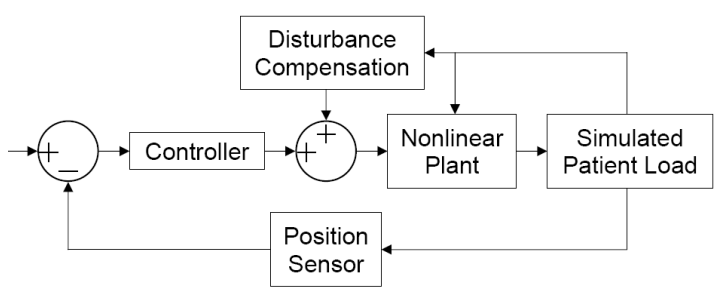

Figure 1. OS actuator model

The Zrs identification model (Figure 2), which was an extension of the actuator model, was used to dynamically characterize the actuator's behaviour during oscillations with simultaneous patient breathing and to identify patient Zrs. This model was constructed by adding various input perturbations, simulated and actual patient breathing noise, and a $\mathrm{Zrs}$ calculation algorithm. With the Zrs identification model, it was possible to guide the redesign while still meeting the performance criteria. The stroke volume needed to produce the required pressure oscillations could be determined by monitoring the pressure and flow outputs of the Zrs identification model in response to a specific input perturbation.

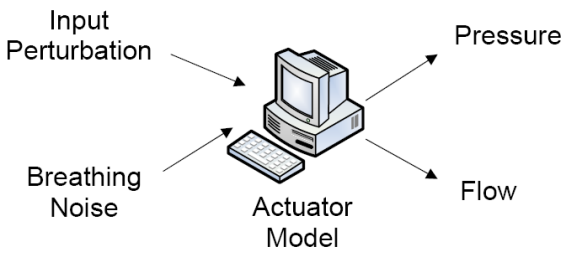

Figure 2. Zrs identification model 


\section{Results}

Results from system modeling and device design are described in the following sections.

\subsection{System Modeling}

Modeling actuator movement as well as simulating actuator behaviour during simultaneous patient breathing were necessary to determine and optimize design parameters such as control variables, stroke volume, and pressure oscillation amplitudes.

The controller employed in the actuator model was a proportional derivative (PD) controller. In choosing the controller, the aim was to avoid steady state error. Integrative control would ideally have zero steady state error. However, since the plant already had a pole at zero (from an integrator), adding another pole would make the system unstable, and thus integrative control was not possible. Tuning of the control parameters was another area of focus. An iterative process was used in which control parameter combinations were tested to achieve maximal rise time and minimal overshoot responses to a step input. An example of this is shown in Figure 3 where the numerator time constant of the PD controller is varied. Based on plots similar to this, optimal control parameters were chosen. It is important to note that detailed optimization was not carried out, since final optimization of the controller would always have to be performed by testing the real system. The parameters found here however, represented a suitable starting point for the final optimization.

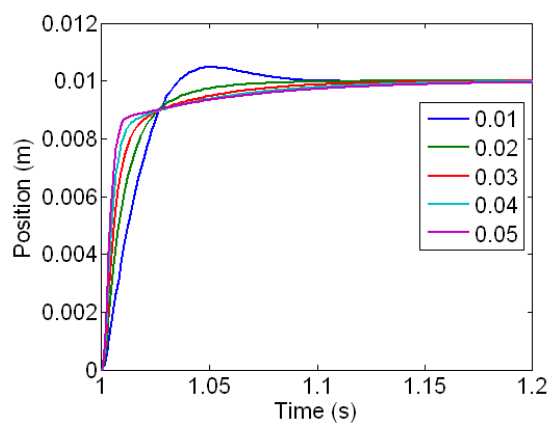

Figure 3. Step responses for varying numerator time constants.

In addition to using the Zrs identification model to accurately determine Zrs to various types and amounts of breathing it was also used to determine the needed input perturbation amplitude required to achieve a 2 $\mathrm{cmH}_{2} \mathrm{O}$ peak-to-peak pressure oscillation in the actuator. Thus, it was possible to determine the needed stroke volume of $30-35 \mathrm{ml}$. Stroke volume dictates the stroke length and therefore the overall length needed for the device.

\subsection{Device Design}

The major mechanical components of the OS included the linear motor, PT, position sensor, and pressure sensor. These major components constituted a total of 18 custom designed and machined parts. Each component is discussed individually below.

\subsubsection{Linear motor}

A linear motor was designed as shown in Figure 4. In the OS, the current was passed through two coils (Transfab TMS, Boucherville, QC, Canada) wound around a bobbin with current flowing in opposite directions in each coil. A rare earth permanent magnet (Dura Magnetics, Sylvania, OH, USA), was used to create a magnetic field that passed from the magnet to the surrounding pole plates, which directed the magnetic flux perpendicularly across the coil to the core, thereby enclosing the bobbin and completing the magnetic circuit.

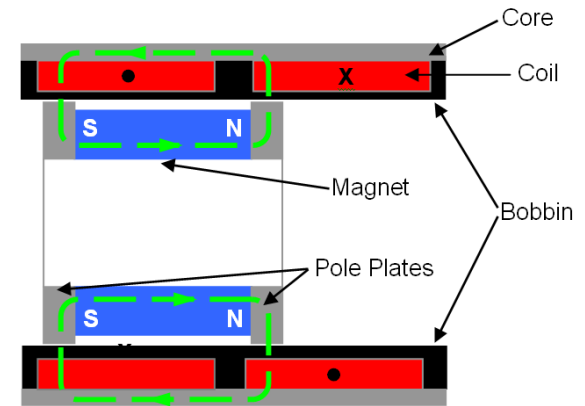

Figure 4. Magnetic circuit for the OS linear motor: The $\mathrm{X}$ represents current flowing through the coil into the page and $\bullet$ represents current flowing through the coil out of the page. The magnetic field travels from the north pole of the magnet, redirected at $90^{\circ}$ by the pole plate, through the coil, along the core, to the other pole plate and finally to the south pole of the magnet.

Additionally to provide stable motion, the actuator moved on parallel rods using low friction bearings. Friction experiments showed it to have a very low coefficient of static friction, 0.3, which was comparable to Teflon at 0.2 . An effort was made to make each part as light as possible. This meant using plastic components wherever applicable and shaving of areas that were not needed for functionality. Attention was also paid to minimizing dead space. Therefore, the piston was designed to always oscillate at the front of the device if the entire stroke length was not being 
used. Furthermore, during oscillation, since air is mixed continuously, dead space is dramatically reduced.

In selecting the magnet, it was necessary to choose one that would make the most efficient motor possible while keeping the cost at a reasonable level. To determine the appropriate magnet dimensions, Matlab was used to approximate the mass of the piston and determine the amount of force necessary to oscillate it at the highest frequency. In order for the piston to move, the generated force $\left(\mathrm{F}_{\text {gen }}\right)$ from the magnet must be greater than the force required $\left(\mathrm{F}_{\text {req }}\right)$ to accelerate the piston mass and the opposing force of friction, according to equation 3.1,

$$
F_{\text {gen }}>F_{\text {req }}=m a+\mu m g
$$

where the piston mass (m) was approximated from mechanical autoCAD drawings and densities of materials, and acceleration (a) was the acceleration of the piston at the highest frequency of interest $(48 \mathrm{~Hz})$. The opposing force of friction was calculated based on the static coefficient of friction $(\mu)$, experimentally determined as 0.3 for Rulon 641 bearings (McMaster Carr Inc., USA), and the acceleration due to gravity (g $=9.8 \mathrm{~m} / \mathrm{s}^{2}$ ).

The wire gauge, type, and number of layers in the bobbin were selected based on the number of effective windings (n) needed and the resistive and inductive properties of the motor. Using the model, we found that decreasing coil resistance increased bandwidth, and effects of altering inductance through the number of turns were minor. We optimized the windings to produce the maximum bandwidth according to the simulation. A bandwidth of $11 \mathrm{~Hz}$ could be achieved for large amplitude perturbations (15 - $35 \mathrm{ml}$ ) with 30 $\mathrm{Hz}$ achievable for smaller amplitudes.

The steel core was designed to be as thin as possible for weight conservation but also thick enough to ensure that all magnetic field lines could be contained. To prevent saturation and therefore loss of the magnetic field and assuming that all flux $(\Phi)$ was carried by the core, the required cross-sectional area (A) could be computed from equation 3.2 for a given $\mathrm{B}$.

$$
\Phi=B A
$$

The core was composed of a low carbon steel (ANSI 1008) that saturates at $B=1.2 \mathrm{~T}$. Using a small safety factor, the core thickness was chosen to ensure that $\mathrm{B}$ was less than $1 \mathrm{~T}$.

\subsubsection{Pneumotachograph (PT) design}

A PT that maintained a linear resistance of $<1$ $\mathrm{cmH}_{2} \mathrm{O} / \mathrm{L} / \mathrm{s}$ at tidal breathing for FOT and $<1.5$ $\mathrm{cmH}_{2} \mathrm{O} / \mathrm{L} / \mathrm{s}$ at $14 \mathrm{~L} / \mathrm{s}$ for spirometry and was heated was desired $[9,16]$.
Screen mesh PTs, which are found in the majority of commercially available flow PTs, where chosen for this design as orifice and groove type PTs were both highly resistive and nonlinear (Figure 5). By changing the diameter of the PT and density of the mesh, the required resistances could be achieved. In addition, the pressure-flow profiles were much more linear as there was a $98 \%$ drop in the second order nonlinearity term compared to the orifice type PTs.

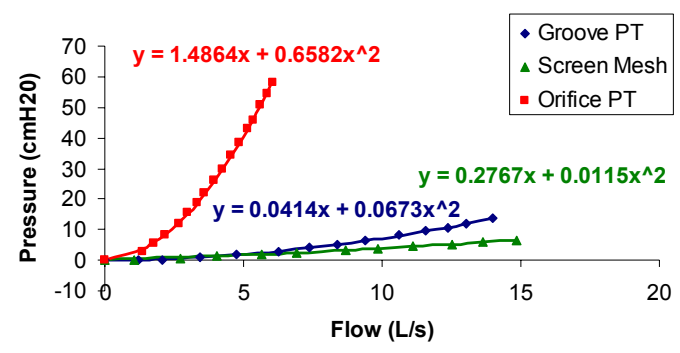

(a)

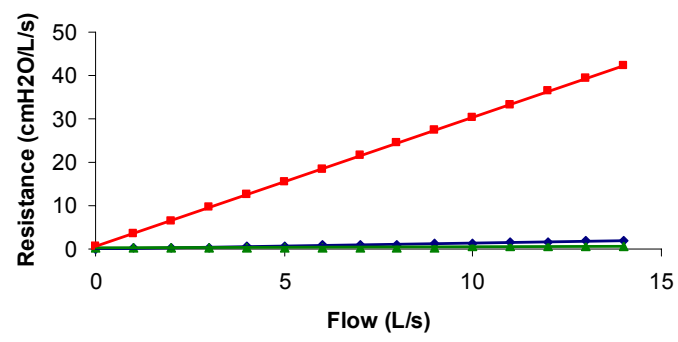

(b)

Figure 5. (a) The orifice PT was the most nonlinear as it exhibited the highest second order term. The screen mesh PT was the most linear with the second order term close to zero. (b) Ideally, a resistance that was as independent of flow as possible was desired. In other words, the resistance would be constant between low flows (for FOT) and high flows (for spirometry). The screen mesh had the least flow dependent resistance and complied with American Thoracic Society standards $[9,16]$.

The mesh was heated by using a highly resistive wire such as nickel-chromium that transferred heat to the mesh once a current was applied. The particular wire was chosen based on its power dissipation and ability to heat the mesh in the actuator adequately with minimal power $(<5 \mathrm{~W})$. A thermistor was incorporated to permit regulated temperature of the mesh to a sufficient constant temperature to prevent condensation.

\subsubsection{Position sensing design}

The OS required a method of measuring the position of the piston for actuator control and for determining $\mathrm{Zrs}$. 
Position was converted via the piston area into volume, then the derivative of volume was taken to give the flow. An optical positioning system was chosen as it was found to be cost effective, accurate, and small enough not to block the flow path or increase the overall size of the device.

The optical positioning system consisted of an infrared light produced by a laser diode (SV5637-001, Richardson Electronics Ltd., Montreal, QC, Canada) that reflected off of a tilted mirror placed on the rear pole plate and shined on a position sensing detector (PSD) located on the back plate. The PSD (1L10, ONTRACK Photonics Inc., Lake Forest, CA, USA) accurately detects the centroid of a light spot to micrometer accuracy and produces a voltage that linearly relates to the centroid position. Using triangulation, as shown in Figure 6, the angle of the mirror and position of the laser and PSD were adjusted such that the laser could reflect off of the mirror while the piston was at the extreme forward and back position and still contact the PSD. To allow sufficient safely margin, the laser, mirror, and PSD were aligned so that $80 \%$ of the active PSD area was being utilized.

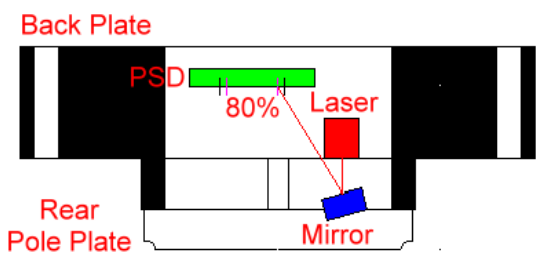

Figure 6. The infrared laser attached to the back plate reflects off of an angled mirror on the rear pole plate and incidents the PSD on the back plate. As the piston moves from back to front, the laser spot moves from left to right along the PSD. The inner marks on the PSD represent $80 \%$ of the active area and the outer marks represent the absolute edges of the active region.

A custom ground circular mirror with a highly reflective gold surface (ME05-M01, Novaphase Inc., Newton, NJ, USA) was placed on the edge of the rear pole plate. The gold coating ensured maximum reflectivity in the infrared wavelength range. The mirror was tilted at a precise $15.5^{\circ}$ angle needed to ensure the laser beam would incident $80 \%$ of the active PSD region. Trigonometric calculations were also performed to determine the maximum error of the position sensor due to piston tilt. Based on the maximum gap, including tolerances, between the linear rods and bearings and the left-right maximal pivoting of the actuator in place, an error of up to $0.17 \mathrm{~mm}$ could occur, which may cause instability of motor position feedback control. However, it was felt that because the two rods and four bearings would have to have the worst case tolerances in the proper orientation simultaneously, such pivoting would be unlikely and the error would be much less.

\subsubsection{Pressure sensing design}

A pressure transducer (5INCH-D1DIP-MV- MINI, All-Sensors Inc., Morgan Hill, CA, USA) to measure airway pressure (for FOT) and another to measure flow (for spirometry) were required. In FOT mode, airway pressure relative to atmosphere was measured from a pressure tap in front of the PT. Flow could then be calculated from piston motion and knowledge of the proximal OS device impedance using a compensation algorithm as described in Schuessler et al. [17].

\section{Discussion and Conclusion}

Using the models and a design process focussed on minimizing dead space and meeting our target pressure and flows, a novel FOT device was developed. The OS was designed to offer stable actuator movement that delivered oscillatory flow at the patient's airway opening and recorded pressure and position signals in real time. Using these signals, Zrs could then be calculated and its variation with time examined.

The user friendly device was compact, taking up $555 \mathrm{~cm}^{3}$ of space, and lightweight $(1 \mathrm{~kg})$. The OS integrated a heated screen mesh PT into the center of the piston, thereby solving issues of PT resistance and nonlinearity as well as condensation accumulation. Through the use of linear rods and bearings, piston play and rotation were eliminated, allowing the linear motor to be stably controlled between 0 - $48 \mathrm{~Hz}$. Airway pressure and the pressure drop across the PT could be measured using two miniature pressure transducers. Position of the piston was determined with an optical positioning system. The flow through design enabled patients to breathe fresh air during measurements, with only $60 \mathrm{ml}$ of dead space. The device was compact and designed with an external appearance that was easy to use and operate (Figure 7).

A custom designed stand will be developed in the future for FOT use and for patients who cannot use the device in a hand-held fashion. In addition, the PT could be easily changed depending on the application and patient size. The inline bacterial filter was successfully designed into the mouthpiece as proof-of-concept that an easy to remove, moulded, disposable mouthpiece and filter could be manufactured in the future.

This report demonstrates an effective protocol for the design of a new medical device. Using computer simulations, the design of the OS was approached using quantitative methods. Modeling work showed the required stroke volume which in turn dictated many of 
the physical dimensions necessary for mechanical design. The computational models were also essential in assessing the affects of design parameters, such as piston diameter and mass, and for optimizing control parameters. By altering design parameters in silico, their effects on the system could be assessed and appropriate constraints could be placed on the design.

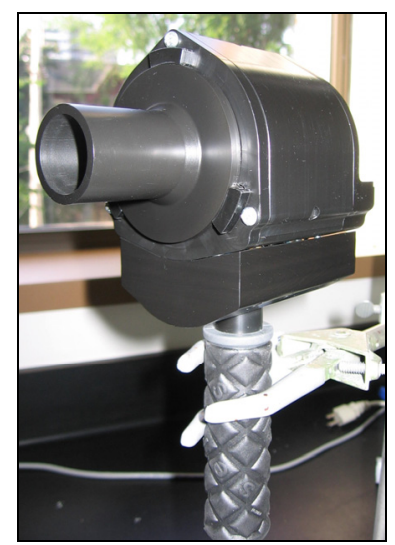

Figure 7. The completed OS

The OS has large potential as a suitable medical device for use by researchers and clinicians. It would help in the diagnosis and monitoring of patients with asthma and other pulmonary disorders. Through the use of FOT, true objective measures of respiratory impedance could be determined in patients across all age groups, as well as paralyzed or otherwise infirm individuals. Development of this device was particularly important as no commercially available device offers measurements of spirometry, FOT, and variability of airway caliber in a portable and light weight hand-held device.

\section{Acknowledgements}

The authors would like to recognize the contributions of Ben Urovitch and Thomas F. Schuessler of SCIREQ Inc., Montreal, Q.C. as well as Victoria de Lange, Jenni Chancey, Swati Bhatawadekar, and Frank Horvath of Dalhousie University. This project was funded by the Nova Scotia Lung Association, Natural Science and Engineering Research Council of Canada (NSERC), and Association of Universities and Colleges of Canada (AUCC).

\section{References}

[1] "Asthma Facts and Statistics [online]," The Canadian Lung Association. Available: http:/www.lung.ca/asthma. [2] A. J. Woolcock and J. K. Peat, "Epidemiology of bronchial hyperresponsiveness," Clin Rev Allergy, vol. 7, pp. 245-256, 1989.
[3] "Worldwide variation in prevalence of symptoms of asthma, allergic rhinoconjunctivitis and ectopic eczema: ISAAC," Lancet, vol. 351, pp. 1225-1232, 1998.

[4] M. Masoli, D. Fabian, S. Holt, and R. Beasley, "Initiative for Asthma," 2004.

[5] "Standardization of Spirometry, 1994 Update. American Thoracic Society," American Journal of Respiratory and Critical Care Medicine, vol. 152, pp. 1107-1136, 1995.

[6] L. P. Boulet, A. Becker, D. Berube, R. Beveridge, and P. Ernst, "Canadian asthma consensus report," Canadian Medical Association Journal, vol. 161, pp. S1-62, 1999.

[7] M. H. Grol, J. Gerritsen, and D. S. Postma, "Asthma: From childhood to adulthood," Allergy, vol. 51, pp. 855-869, 1996.

[8] S. Kanengiser and A. J. Dozer, "Forced expiratory maneuvers in children aged 3 to 5 years," Pediatric

Pulmonology, vol. 18, pp. 144-149, 1994.

[9] E. Oostveen, D. Macleod, H. Lorino, R. Farre, Z. Hantos, K. Desager, and F. Marchal, "The forced oscillation technique in clincial practice: methodology, recommendations and future developments," European Respiratory Journal, vol. 22, pp. 1026-1041, 2003.

[10] F. Gimeno, L. T. v. d. Weele, G. H. Koeter, J. G. d. Monchy, and R. v. Altena, "Variability of forced oscillation (Siemens Siregnost FD 5) measurements of total respiratory resistance in patients and healthy subjects," Ann Allergy, vol. 71, pp. 56-60, 1993.

[11] M. D. Goldman, R. Carter, R. Klein, G. Fritz, B. Carter, and P. Pachucki, "Within-and between-day variability of respiratory impedance using impulse oscillometry in adolescent asthmatics," Pediatric Pulmonology, vol. 34, pp. 312-319, 2002.

[12] K. L. Timonen, J. T. Randell, R. O. Salonen, and J. Pekkanen, "Short-term variations in oscillatory and spirometric lung function indices among school children," European Respiratory Journal, vol. 10, pp. 82-87, 1997. [13] M. Cauberghs and K. V. d. Woestijine, "Changes of respiratory input impedance during breathing in humans," Journal of Applied Physiology, vol. 73, pp. 2355-2362, 1992. [14] R. Peslin, Y. Ying, C. Gallina, and C. Duvivier, "Within-breath variations of forced oscillation resistance in healthy subjects," European Respiratory Journal, vol. 5, pp. 86-92, 1992.

[15] C. A. Lall, N. Cheng, P. Hernandez, P. T. Pianosi, Z. Dali, A. Abouzied, and G. N. Maksym, "Airway resistance variability and response to bronchodilator in children with asthma," European Respiratory Journal, vol. 30, pp. 260268, 2007.

[16] M. R. Miller, J. Hankinson, V. Brusasco, F. Burgos, R. Casaburi, A. Coates, R. Crapo, P. Enright, C. P. M. v. d. Grinten, P. Gustafsson, R. Jensen, D. C. Johnson, N. MacIntyre, R. McKay, D. Navajas, O. F. Pedersen, R. Pellegrino, G. Viegi, and J. Wanger, "Standardisation of spirometry," European Respiratory Journal, vol. 26, pp. 319338, 2005.

[17] T. F. Schuessler and J. H. T. Bates, "A computercontrolled research ventilator for small animals: design and evaluation," IEEE Transactions on Biomedical Engineering, vol. 42, pp. 860-866, 1995. 